\section{Acknowledgments.}

Financial support. No financial support was provided relevant to this article.

Conflicts of interest. All authors report no conflicts of interest relevant to this article.

\section{References}

1. Hayes SC, Davies PS, Parker TW, Bashford J, Green A. Role of a mixed type, moderate intensity exercise programme after peripheral blood stem cell transplantation. Br J Sports Med 2004;38:304-309.

2. El-Jawahri A. R., Vandusen H. B., Traeger L. N. et al. Quality of life and mood predict posttraumatic stress disorder after hematopoietic stem cell transplantation. Cancer 2016;122:806-812.

3. Freifeld AG, Bow EJ, Sepkowitz KA, et al. Clinical practice guideline for the use of antimicrobial agents in neutropenic patients with cancer: 2010 update by the Infectious Diseases Society of America. Clin Infec Dis 2011;52:e56-e93.

4. Tomblyn M, Chiller T, Einsele H, et al. Guidelines for preventing infectious complications among hematopoietic cell transplantation recipients: a global perspective. Biol Blood Marrow Transpl 2009;15:1143-1238.
5. Sokol KA, De La Vega-Diaz I, Edmondson-Martin K, et al. Masks for prevention of respiratory viruses on the BMT unit: results of a quality initiative. Transpl Infect Dis 2016;18:965-967.

6. Hayes-Lattin B, Leis JF, Maziarz RT. Isolation in the allogeneic transplant environment: How protective is it? Bone Marrow Transpl 2005;36: 373-381.

7. Interim guidance on the use of influenza antiviral agents during the 20102011 influenza season. US Centers for Disease Control and Prevention website. http://www.cdc.gov/flu/professionals/antivirals/guidance/summary.htm. Published 2012. Accessed on March 13, 2018.

8. American Academy of Pediatrics. Respiratory syncytial virus. In: Red Book: 2015 Report of the Committee on Infectious Diseases, $30^{\text {th }}$ Edition, Kimberlin DW, Brady MT, Jackson MA, Long SS, eds. Elk Grove Village, IL: American Academy of Pediatrics; 2015.

9. Centers for Disease Control and Prevention (CDC). Outbreaks of human metapneumovirus in two skilled nursing facilities-West Virginia and Idaho, 2011-2012, MMWR Morb Mortal Wkly Rep 2013;62:909-913.

10. American Academy of Pediatrics. Parainfluenza viral infections. In: Red Book: 2015 Report of the Committee on Infectious Diseases, $30^{\text {th }}$ Edition, Kimberlin DW, Brady MT, Jackson MA, Long SS, editors. Elk Grove Village, IL: American Academy of Pediatrics; 2015.

\title{
Impact of elimination of contact precautions on noninfectious adverse events among MRSA and VRE patients
}

\author{
Sumanth Gandra MD ${ }^{1,2}$, Constance M. Barysauskas $\mathrm{MS}^{3}$, Deborah A. Mack RN ${ }^{4}$, Bruce Barton PhD ${ }^{5}$, Robert Finberg \\ $M D^{6}$ and Richard T. Ellison $M D^{6}$ \\ ${ }^{1}$ Medical Microbiology Fellow, Department of Pathology, University of Chicago, Chicago, Illinois, ${ }^{2}$ NorthShore University Health System, Evanston Hospital, \\ Evanston, Illinois, ${ }^{3}$ Veristat, Southborough, Massachusetts, ${ }^{4}$ Infection Control Department, University of Massachusetts Medical Center, Worcester, \\ Massachusetts, ${ }^{5}$ Quantitative Health Sciences, University of Massachusetts Medical School, Worcester, Massachusetts and and ${ }^{6}$ Division of Infectious Disease \\ and Immunology, University of Massachusetts Medical School, Worcester, Massachusetts
}

To the Editor-We read with great interest the article by Martin et $\mathrm{al}^{1}$ published online in May 2018 in Infection Control and Hospital Epidemiology. ${ }^{1}$ We previously reported on the impact of elimination of contact precautions (CP) in methicillin-resistant Staphylococcus aureus (MRSA) and vancomycin-resistant enterococci (VRE) patients on noninfectious complications, although our analysis was limited to falls and pressure ulcers. ${ }^{2}$ Our findings differed from those of Martin et al; we observed no statistically significant difference in the rate of falls or pressure ulcers among MRSA/VRE patients in the years before and after eliminating CP. The rate of falls among MRSA/VRE patients in the year before eliminating CP was 4.57 per 1,000 patient days, and it was 4.82 per 1,000 patient days in the year after eliminating contact precautions $(P=074)$. Similarly, the rate of pressure ulcers in the year before eliminating $\mathrm{CP}$ was 4.87 per 1,000 patient days, and it was 4.17 per 1,000 patient days in the year after eliminating contact precautions $(P=.33)$.

Martin et al report a significant drop in the number of noninfectious adverse events among MRSA/VRE patients in the year

Author for correspondence: Sumanth Gandra MD, Room 1917, Evanston Hospital, 2650 Ridge Avenue, Evanston, IL 60201. E-mail: gandrasatyam@gmail.com

Cite this article: Gandra S, et al. (2018). Impact of elimination of contact precautions on noninfectious adverse events among MRSA and VRE patients. Infection Control \& Hospital Epidemiology 2018, 39, 1272-1273. doi: 10.1017/ice.2018.204 after eliminating CP (21.4 per 1000 admissions vs 6.08 per 1000 admissions; $P<.001$ ). In contrast to our study, the study summarized by Martin et al not only included falls and pressure ulcers but also hemorrhage, postoperative respiratory failure, wound dehiscence, and pulmonary embolism or deep vein thrombosis. Although the composite index of all noninfectious adverse events showed a significant drop, the authors did not present a breakdown by individual adverse events in MRSA/VRE and nonMRSA/VRE patients.

We reported 2 additional important findings in our study. First, MRSA/VRE patients had a statistically significant higher Charlson comorbidity index (CCI) compared with non-MRSA/VRE patients (mean CCI, 3.32 vs $2.75 ; P=.002$ ). This was not examined by Martin et al. Second, compared to non-MRSA/VRE patients, we found that MRSA/VRE patients had significantly higher rates of falls (4.57 per 1,000 patient days vs 2.04 per 1,000 patient days) and pressure ulcers (4.87 per 1,000 patient days vs 1.22 per 1,000 patient days), both in the year before and in the year after eliminating CP. Based on figure 2 from Martin EM et al, the rate of noninfectious adverse events were much higher in MRSA/VRE patients than in non-MRSA/VRE patients in the year prior to elimination of CP. However, in the year thereafter, there seems to be no difference.

The reason for the discrepancy in the findings between the 2 studies is unclear. However, we have identified 2 differences between 
the studies. First, the rate of individual adverse events between the MRSA/VRE and non-MRSA/VRE patients in Martin et al are not included. Second, for rate calculation, we calculated the rate per 1,000 patient days, whereas Martin et al considered the rate per 1,000 admissions. Whether this had any influence on outcomes is unknown.

Similar to the previous study by Martin et $\mathrm{al}^{3}$ that indicated no change in the healthcare associated infection (HAI) rates of MRSA/VRE after elimination of $\mathrm{CP}$, we also reported no significant change in HAI rates in MRSA/VRE patients after eliminating $\mathrm{CP}$ in our study. ${ }^{2}$ Thus, eliminating CP for MRSA/VRE patients is not associated with increased HAI rates with MRSA/ VRE and could improve patient safety outcomes. Our observation that MRSA/VRE patients are at higher risk of noninfectious adverse events argues the need for serious consideration of eliminating CP among MRSA/VRE patients.
Financial support. No financial support was provided relevant to this article.

Conflicts of interest. All authors report no conflicts of interest relevant to this article.

\section{References}

1. Martin EM, Bryant B, Grogan TR, et al. Noninfectious hospital adverse events decline after elimination of contact precautions for MRSA and VRE. Infect Control Hosp Epidemiol 2018. Published online May 10, 2018, p. 1-9.

2. Gandra S, Barysauskas C, Mack DA, Barton B, Finberg R, Ellison RT III. Impact of contact precautions on falls, pressure ulcers and transmission of MRSA and VRE in hospitalized patients. J Hosp Infect 2014; 88:170-176.

3. Martin EM, Russell D, Rubin Z, et al. Elimination of routine contact precautions for endemic methicillin-resistant Staphylococcus aureus and vancomycin-resistant Enterococcus: a retrospective quasi-experimental study. Infect Control Hosp Epidemiol 2016; 37:1323-1330.

\title{
Collaboration for containment: Detection of OXA-23-like carbapenamase-producing Acinetobacter baumannii in Colorado
}

\author{
Heather L. Young $\mathrm{MD}^{1}$, Caroline Croyle $\mathrm{MPH}^{2}$, Sarah J. Janelle MPH, $\mathrm{CIC}^{3}$, Bryan C. Knepper MPH, MSc, $\mathrm{CIC}^{2}$, \\ Jennifer Kurtz BSN, MS ${ }^{2}$, Amber Miller MSN, CIC, CHFM ${ }^{4}$, Sara M. Reese PhD, MPH, $\mathrm{CIC}^{5}$, Kyle Schutz MSc ${ }^{3}$ and \\ Wendy M. Bamberg MD ${ }^{3}$ \\ ${ }^{1}$ Department of Medicine, Denver Health Medical Center and University of Colorado School of Medicine, Denver, Colorado, ${ }^{2}$ Department of Patient Safety and \\ Quality, Denver Health Medical Center, Denver, Colorado, ${ }^{3}$ Colorado Department of Public Health and Environment, Denver, Colorado, ${ }^{4}$ Department of Planning \\ and Construction, University of Colorado Health, Aurora, Colorado and ${ }^{5}$ Department of Quality. Swedish Medical Center, Denver, Colorado
}

To the Editor-Multidrug-resistant Acinetobacter baumannii (MDR-AB) is an aggressive pathogen often transmitted in healthcare facilities. Critically ill patients are at highest risk, particularly those with recent surgery, prolonged ventilation, and exposure to broad spectrum antibiotics. ${ }^{1}$ Containment of MDR$A B$ requires early identification and multifaceted interventions.

MDR-AB strains that are resistant to carbapenems present additional containment issues because plasmid-mediated carbapenemase production is a common resistance mechanism. ${ }^{2}$ Given its importance as an emerging antimicrobial-resistant pathogen, many public health departments, including the Denver metropolitan region in Colorado, require carbapenem-resistant Acinetobacter baumannii (CRAB) to be reported.

Between December 2017 and February 2018, Denver Health Medical Center (DHMC) detected 2 inpatients with carbapenemaseproducing CRAB isolates in urine. Prior to these cases, no previous $\mathrm{CRAB}$ isolates in Colorado had been characterized as carbapenemase-producing organisms. DHMC and the Colorado Department of Public Health and Environment (CDPHE) collaborated to determine epidemiologic and molecular relatedness of the

Author for correspondence: Heather Young MD, Denver Health Medical Center, 601 Broadway, MC 4000, Denver CO 80204. E-mail: Heather.Young2@dhha.org

ADDITIONAL PRESENTATION. This work has been accepted as a poster abstract at the ID Week conference in San Francisco, California, in October 2018.

Cite this article: Young HL. et al. (2018). Collaboration for containment: Detection of OXA-23-like carbapenamase-producing Acinetobacter baumannii in Colorado. Infection Control \& Hospital Epidemiology. 2018, 39, 1273-1274. doi: 10.1017/ice.2018.202 isolates, as well as to investigate healthcare infection control measures.

DHMC is a 555-bed safety net teaching hospital and level 1 trauma center located in Denver, Colorado. DHMC previously reported an MDR-AB outbreak between 2004 and 2005, ${ }^{3}$ and these MDR-AB isolates retained carbapenem susceptibility. Regionally, CRAB is unusual in the Denver metropolitan region, with 2-13 cases reported from sterile body sites and urine per year since 2013.

The CDPHE epidemiologists and DHMC infection preventionists performed surveillance for additional cases that met the case definition. CRAB isolates were defined as those that had a minimum inhibitory concentration (MIC) to at least 1 carbapenem in the intermediate or resistant range. Investigators reviewed medical records for common hospital locations, medical equipment, procedures, and staff members. Infection preventionists observed practices among shared staff members. Pulsed-field gel electrophoresis (PFGE) was performed at the CDPHE laboratory, while antimicrobial susceptibility and carbapenemase testing was performed at the Centers for Disease Control and Prevention (CDC).

The epidemiologic investigation revealed several similarities. Patient 1 was a 59-year-old male with diabetes mellitus and spina bifida, while patient 2 was a 23 -year-old male with lymphangiomatosis and resulting T6 paraplegia. Both patients had neurogenic bladders managed by suprapubic catheters, stage 4 decubitus ulcers, recent surgery, and extensive antibiotic exposure. Neither had recently traveled outside of Colorado nor received a carbapenem in the prior 6 months. CRAB was detected from urine 